\title{
ANÁLISE DA CINÉTICA E AJUSTES DE MODELOS MATEMÁTICOS AOS DADOS DE SECAGEM DO BAGAÇO DO MARACUJÁ-AMARELO
}

\author{
Maraisa Lopes de Menezes ${ }^{I}$ \\ Ana Paula Ströher \\ Nehemias Curvelo Pereira ${ }^{1}$ \\ Sueli Teresa Davantel de Barros ${ }^{l}$
}

\begin{abstract}
Resumo - Este trabalho teve por objetivo analisar a secagem convectiva em leito fixo do bagaço do maracujá-amarelo. Primeiramente, determinou-se a umidade inicial do bagaço em estufa, durante $24 \mathrm{~h}$ a $105^{\circ} \mathrm{C} \pm 3^{\circ} \mathrm{C}$. Nos ensaios de secagem, foram coletados os valores de massa do bagaço em função do tempo, utilizando-se um secador convectivo, operado em quatro temperaturas $\left(35,45,55\right.$ e $\left.65^{\circ} \mathrm{C}\right)$ e três velocidades do ar de 0,8 , 1,0 e $1,3 \mathrm{~m} . \mathrm{s}^{-1}$. Por meio das curvas de secagem e da taxa de secagem, observou-se que a temperatura exerce grande influência na secagem do bagaço, pois quando se aumenta a temperatura ocorre uma diminuição do tempo de secagem e um consequente aumento na taxa e na difusividade efetiva. As curvas de secagem e de taxa de secagem foram ajustadas aos modelos propostos por Page e Motta Lima e colaboradores e aos modelos propostos por Hogdes e Toffoli, respectivamente. Outros modelos matemáticos também foram ajustados para cada secagem, tais como: Exponencial Simples, Page, Henderson e Pabis, Logaritmo, Exponencial de Dois Termos e Wang e Singh. Por meio dos resultados foi verificado que o modelo de Page foi o que melhor se ajustou aos dados experimentais de secagem com base nos maiores valores do $\mathrm{R}^{2}$ e Teste $\mathrm{F}$ e no menor valor da raiz do erro médio.
\end{abstract}

Palavras-chave: Secagem convectiva, curvas de secagem, ajuste de modelos matemáticos.

Abstract - This work aimed to analyze the convective drying in fixed bed of yellow passion fruit bagasse. Firstly, the initial bagasse moisture was determined in stove for 24 hours at $105^{\circ} \mathrm{C} \pm 3{ }^{\circ} \mathrm{C}$. It was also collected the mass values of the bagasse in function of time, by using a convective drier, operated at four different temperatures $\left(35,45,55\right.$ and $65^{\circ} \mathrm{C}$ ) and three air flow speeds at $0.8,1.0$ and $1.3 \mathrm{~m} . \mathrm{s}^{-1}$. By means of the drying curves and the drying rate, it could be observed that the temperature highly influences on the bagasse drying process, once as the temperatures increase, the drying time decreases, and then the drying rate and effective diffusivity becomes higher. The drying curves and the drying rate were adjusted to the models proposed by Page and Motta Lima and collaborators and the models proposed by Hogdes and Toffoli, respectively. Other mathematical models were also adjusted for each drying process, such as: Simple Exponential, Page, Henderson and Pabis, Logarithm, two-term exponential model and Wang and Singh. Through the results showed that the Page model was the best fit to the experimental data of drying based on the highest values of $\mathrm{R}^{2}$ and $\mathrm{F}$ Test and the lowest root mean error.

Keywords: Convective drying, drying curves, mathematical models adjustments.

1 Programa de Pós-graduação Stricto Sensu em Engenharia Química da Universidade Estadual de Maringá - UEM. Av. Colombo, 5790. Jd. Universitário. CEP: 87020-900. Email: maraisalm@hotmail.com 


\section{INTRODUÇÁO}

O Brasil é considerado o maior produtor mundial de maracujá, apresentando uma área plantada de aproximadamente 33 mil hectares, principalmente nos estados da Bahia, São Paulo e Sergipe, sendo responsável por cerca de $90 \%$ da produção mundial, seguido pelo Peru, Venezuela, África do Sul, Sri Lanka e Austrália (Ferrari et al., 2004).

A família Passifloraceae é amplamente distribuída nos trópicos e também nas regióes temperadas quentes, em especial, da América e África, sendo que grande parte das espécies, cerca de 400, é do gênero Passiflora que se distribui principalmente na América (apenas 20 ocorrem na Índia, China, Sudeste Asiático, Austrália, ilhas da Oceania e regiōes vizinhas). $\mathrm{O}$ segundo maior gênero, Adenia, apresenta cerca de 100 espécies (Cronquist, 1981), com ocorrência na África, Ásia e Oceania. Entre os outros gêneros apenas Basananthe apresenta mais do que 20 espécies, sendo sete gêneros monotípicos (Escobar, 1988). No Neotrópico, ocorrem cinco gêneros e quase 400 espécies e, no Brasil, ocorrem cerca de 130 (Bernacci et al., 2003).

As espécies mais cultivadas no Brasil e no mundo são: maracujá-amarelo, maracujá-roxo $(P$. edulis Sims) e, também, o maracujá-doce (P. alata Curtis) (Bernacci et al., 2003).

Um dos subprodutos da indústria de extração de suco de maracujá com menor valor agregado é o bagaço do maracujá úmido, composto principalmente pelo flavedo (parte com coloraçáo) e albedo (parte branca). Em 2000, 127,7 mil toneladas do fruto foram processadas segundo a Associação das Indústrias processadoras de Frutos Tropicais (ASTN). Isso gerou 51 mil toneladas de casca fresca, que desidratadas gerariam 10,2 mil toneladas de casca seca.

A secagem do bagaço do maracujá proporciona a redução de volume e peso, facilitando o armazenamento e transporte deste material, permitindo desta maneira, agregar valor e desenvolver outras aplicaçóes a este resíduo industrial de baixo custo (Doymaz, 2005).

A secagem de um material sólido qualquer, envolve simultaneamente a transferência de calor e de massa (Mwithiga; Olwal, 2005; Corrêa et al. 2006), e seu comportamento é caracterizado pela análise das mudanças de umidade em função do tempo.

Por meio da secagem, é possível ser determinada a cinética de secagem, a qual procura determinar o comportamento do material sólido que é seco, sendo representada pelas curvas de secagem e de taxa de secagem. O comportamento da curva de secagem de um sólido úmido mediante um fluxo de ar a uma determinada temperatura é sempre o mesmo, segundo Foust et al. (1980). Já a curva da taxa de secagem é obtida derivando-se os dados de umidade em função do tempo de secagem.

A cinética de secagem de vários produtos também pode ser descrita por modelos matemáticos semiempíricos ou puramente empíricos (Madamba, 2003). Os modelos semiempíricos se baseiam na analogia com a Lei de Newton para o resfriamento, aplicada à transferência de massa, enquanto que, os modelos empíricos apresentam uma relação entre o conteúdo médio de umidade e o tempo de secagem e consideram como mecanismo principal a difusão baseada na segunda Lei de Fick (Zanoelo et al., 2007).

Com base no que foi exposto, este trabalho teve por objetivos: analisar o comportamento da secagem convectiva do bagaço do maracujá-amarelo em quatro diferentes temperaturas e três velocidades do fluxo de ar; aplicar modelos matemáticos da literatura aos dados experimentais; selecionar o modelo que melhor representa o comportamento dos dados experimentais.

\section{MATERIAL E MÉTODOS}

\subsection{MATERIAL}

O bagaço do maracujá-amarelo foi obtido numa indústria de polpa de frutas para sucos, localizada em Japurá-PR. Após a coleta da matéria-prima, esta foi triturada em liquidificador e separada em amostras de aproximadamente 500 g. Cada amostra foi colocada em sacos plásticos que depois foram vedados. Os pacotes foram estocados em um congelador, no qual a temperatura foi mantida a $-15 \pm 2{ }^{\circ} \mathrm{C}$.

Todos os ensaios experimentais foram realizados no Laboratório de Processos de Separaçáo I da Universidade Estadual de Maringá.

\subsection{DETERMINAÇÃO DAS CURVAS DE SECAGEM E DE TAXA DE SECAGEM}

Primeiramente, determinou-se a umidade inicial do bagaço do maracujá em estufa a $105 \pm 3{ }^{\circ} \mathrm{C}$ por $24 \mathrm{~h}$.

Para o levantamento dos dados cinéticos necessários no estudo da secagem convectiva do bagaço do maracujá-amarelo, foi utilizado um secador de leito fixo com fluxo ascendente, conforme mostra a Figura 1, sendo os ensaios realizados em 
camada delgada (espessura $=0,005 \mathrm{~m}$ ), utilizando aproximadamente $500 \mathrm{~g}$ de bagaço.

Figura 1- Desenho esquemático do secador convectivo de leito fixo

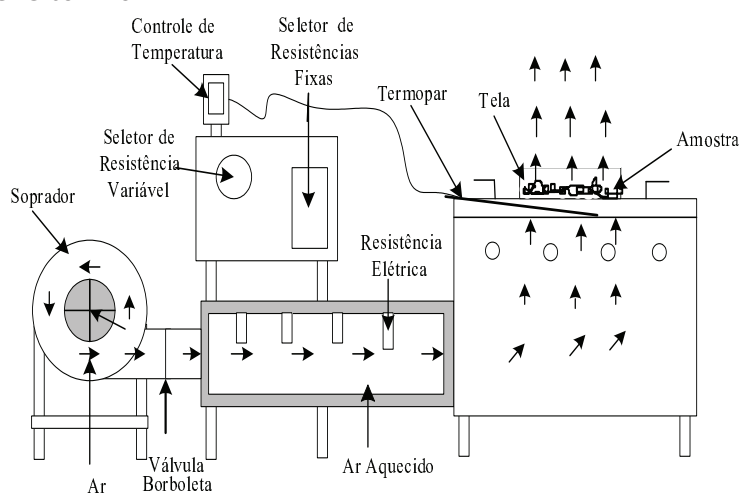

O fluxo de ar utilizado na secagem provém de um ventilador no qual a vazão de ar pode ser ajustada por meio de uma válvula borboleta, sendo que a medida da velocidade do fluxo de ar foi feita por um anemômetro digital portátil (Modelo HH-F10 - precisão: $0,1 \mathrm{~m} . \mathrm{s}^{-1}$ ) posicionado imediatamente acima da bandeja do secador e a medida da umidade ambiente, foi realizada com auxílio de um psicrômetro digital (Modelo THWD - 1 - precisão: $1,0{ }^{\circ} \mathrm{C}$ - temperatura; $3 \%$ - umidade).

Após ser estabelecida a velocidade do fluxo de ar, a temperatura foi variada a partir de resistências elétricas dispostas num aquecedor no qual o ar passa antes de entrar em contato com a matéria-prima. Essas resistências são acionadas, individual ou simultaneamente até que a temperatura desejada seja estabelecida.

Os experimentos foram realizados em duplicata, com variação da velocidade do fluxo de ar $(0,8$; 1,0 e 1,3 m.s. $\left.{ }^{-1}\right)$ e da temperatura $(35,45,55$ e 65 $\left.{ }^{\circ} \mathrm{C}\right)$. Nos primeiros $30 \mathrm{~min}$, pesava-se a bandeja em balança digital (GEHAKA Linha Bg 4000 - precisão: $0,01 \mathrm{~g}$ ) num intervalo de $2 \mathrm{~min}$, em seguida, até $1 \mathrm{~h}$ o intervalo de pesagem passou a ser de $5 \mathrm{~min}$ e de $1 \mathrm{~h}$ a $1 \mathrm{~h} 30 \mathrm{~min}$ de $10 \mathrm{~min}$, depois o intervalo passou a ser de $15 \mathrm{~min}$ até o final da secagem, isto é, até massa constante.

Com esses dados coletados, foram construídas as curvas de secagem e de taxa de secagem. A primeira foi obtida por um gráfico de umidade em função do tempo. A umidade em cada instante foi obtida a partir da Equação 1.

$\mathrm{X}(\mathrm{b} . \mathrm{s})=\frac{\mathrm{M}_{\text {úmida }}-\mathrm{M}_{\text {sec a }}}{\mathrm{M}_{\text {sec a }}}$
Em que: $\mathrm{X}$ (b.s) é a razão entre a massa de água presente na amostra $\left(M_{\text {úmida }}-M_{\text {seca }}\right)$ e a massa de sólido isenta desta umidade $\left(\mathrm{M}_{\text {seca }}\right)$, em um determinado tempo. $\mathrm{M}_{\text {úmida }}$ é a massa da amostra antes da estufa (g) e $\mathrm{M}_{\text {seca }}$ é a massa seca obtida em estufa (g).

Para a análise econômica do processo, visando-se determinar a melhor condição de secagem na qual a amostra apresentasse uma menor umidade de equilíbrio para o armazenamento em um curto tempo de secagem, foi avaliada a potência das resistências necessária para se atingir a temperatura de secagem desejada.

As curvas de taxa de secagem (R) foram obtidas a partir da derivaçáo das respectivas curvas de secagem pelo método numérico $(\Delta X / \Delta t)$, obtendo-se o valor da umidade nos pontos originalmente utilizados na construção das curvas de secagem, conforme Motta Lima et al. (2002).

O procedimento de cálculo da taxa de secagem no ponto i é a seguir detalhado na Equação 2.

$(\Delta \mathrm{X} \Delta \mathrm{t})_{\mathrm{i}-}($ entre i-1 e i $)$ e $(\Delta \mathrm{X} \Delta \mathrm{t})_{\mathrm{i}+}($ entre i e i +1$)$

$(\Delta \mathrm{X} \Delta \mathrm{t})_{\mathrm{i}}=\left[(\Delta \mathrm{X} \Delta \mathrm{t})_{i_{-}}+(\Delta \mathrm{X} \Delta \mathrm{t})_{\mathrm{i}+}\right] 2$

em $\mathrm{X}_{0}=(\Delta \mathrm{X} \Delta \mathrm{t})_{0+}$ ou $(\Delta \mathrm{X} \Delta \mathrm{t})_{\mathrm{i}+}$

em $X_{C}=(\Delta X \Delta t)_{X_{e^{-}}}$

\subsection{DETERMINAÇÃO DO COEFICIENTE DE DIFUSIVIDADE E DA ENERGIA DE ATIVAÇÃO}

Para a determinação da difusividade efetiva, partiu-se da equação de Crank (1975).

$$
Y=\frac{X-X_{e q}}{X_{0}-X_{e q}}=\frac{8}{\pi^{2}} \sum_{n=1}^{\infty} \frac{1}{(2 n-1)^{2}} \exp \left(-\frac{(2 n-1)^{2} \pi^{2} D_{e f f} t}{4 L^{2}}\right)
$$

Em que:

$\mathrm{Y}$ - adimensional de teor de água;

$\mathrm{X}$ - teor de água médio, $\mathrm{kgH}_{2} \mathrm{O} / \mathrm{kgm}_{\text {seca }}$;

$\mathrm{X}_{\text {eq }}$ - teor de água de equilíbrio, $\mathrm{kgH}_{2} \mathrm{O} / \mathrm{kgm}_{\text {seca; }}$;

$\mathrm{X}_{0}$ - teor de água no instante inicial, $\mathrm{kgH}_{2} \mathrm{O} / \mathrm{kgm}_{\text {seca }}$;

$\mathrm{D}_{\text {eff }}$ - coeficiente de difusividade, $\mathrm{m}^{2} / \mathrm{s}$;

$\mathrm{t}$ - tempo, s;

L - comprimento característico, semi-espessura da amostra, m.

Para tempos longos de secagem considera-se $\mathrm{n}=1$ e a Equação 3 pode ser reduzida à apenas o primeiro termo da série, obtendo-se a Equação 4.

$$
X=\frac{8}{\pi^{2}} \exp \left(-\frac{\pi^{2} D_{\text {eff }} t}{4 L^{2}}\right)
$$


Linearizando a Equação 4 e plotando-se o gráfico de $\ln (\mathrm{X})$ em funçáo do tempo obtém-se a inclinação $\left(\mathrm{K}_{0}\right)$ de acordo com a Equação 5 , a partir da qual foi possível determinar a $\mathrm{D}_{\text {eff }}$ para cada condição de secagem.

$$
\mathrm{K}_{0}=\frac{\pi^{2} \mathrm{D}_{\mathrm{eff}}}{4 \mathrm{~L}^{2}}
$$

Em que: L é a metade da espessura da amostra $(\mathrm{m})$.

Pode-se entender a difusividade como a facilidade com que a água é removida do material. Como a difusividade varia conforme mudam as condiçóes de secagem (temperatura e velocidade do fluxo de ar), ela não é intrínseca ao material. Assim, convenciona-se chamá-la de difusividade efetiva (Sousa, 2003). O coeficiente de difusão ( $\left.D_{\text {eff }}\right)$ é uma difusividade efetiva que engloba os efeitos de todos os fenômenos que podem intervir sobre a migração da água, sendo dependente da temperatura, como mostra a Equação 6, utilizada para o cálculo da energia de ativação para cada condição de secagem.

$$
\mathrm{D}_{\text {eff }}=\mathrm{D}_{0} \exp \left(-\frac{\mathrm{E}_{\mathrm{act}}}{\mathrm{R} \mathrm{T}}\right)
$$

Em que: $\mathrm{D}_{0}$ é o fator pré-exponencial da equação de Arrhenius $\left(\mathrm{m}^{2} / \mathrm{s}\right)$, R a constante universal dos gases $(8,314 \mathrm{~J} / \mathrm{mol} \mathrm{K}), \mathrm{T}$ a temperatura absoluta $(\mathrm{K})$ e $\mathrm{E}_{\text {act }}$ a energia de ativação $(\mathrm{kJ} / \mathrm{mol})$.

\subsection{MODELAGEM DO PROCESSO DE SECAGEM}

As curvas generalizadas de secagem são outro meio de avaliar a umidade de uma amostra. Trata-se de curvas que relacionam a umidade adimensionalizada das amostras com uma variável adimensional de tempo.

Alguns autores propuseram em seus trabalhos equaçóes generalizadas para a secagem. Page (1949) propôs o seguinte modelo dado pela Equação 7.

$$
\mathrm{X}_{\mathrm{ad}}=\exp \left(-\mathrm{K}_{1} \mathrm{t}^{\mathrm{a}_{1}}\right)
$$

Posteriormente, Motta Lima et al. (2002) modificaram a Equação 7 obtendo a Equação 8.

$$
X_{a d}=\exp \left(-K_{2} t_{a d}{ }^{\left(a_{2} t_{a d}+b_{2}\right)}\right)+0,01
$$

Nas duas equaçóes apresentadas tem-se que $\mathrm{X}_{\mathrm{ad}}=\mathrm{X} / \mathrm{X}_{0}$, na qual $\mathrm{X}$ é a umidade em dado tempo e $\mathrm{X}_{0}$ é a umidade inicial do bagaço do maracujá; $\mathrm{t}_{\mathrm{ad}}=\mathrm{t} . \mathrm{N}_{\mathrm{C}} / N_{0}$, em que $\mathrm{t}_{\text {ad }}$ é o tempo adimensional, t é o tempo, $\mathrm{N}_{\mathrm{c}}$ é a taxa de secagem constante; $\mathrm{k}_{1}, \mathrm{k}_{2}, \mathrm{a}_{1}, \mathrm{a}_{2}, \mathrm{~b}_{2}$ são parâmetros das equaçóes.

Para as curvas generalizadas de taxa de secagem, Hogdes (1982) propôs:

$$
\mathrm{TSN}=\frac{\mathrm{N}_{\mathrm{T}}}{\mathrm{N}_{\max }}=1-\exp \left(-\left(\frac{\mathrm{X}}{\mathrm{a}_{3}}\right)^{\mathrm{b}_{3}}\right)
$$

Baseado na equação de Hogdes (1982), Toffoli (2005) fez uma variação em seu expoente de forma linear com a umidade e chegou à Equaçáo 10.

$$
\operatorname{TSN}=\frac{\mathrm{N}_{\mathrm{T}}}{\mathrm{N}_{\max }}=1-\exp \left(-\left(\frac{\mathrm{X}}{\mathrm{a}_{4}}\right)^{\left(\mathrm{b}_{4} \mathrm{X}+\mathrm{c}_{4}\right)}\right)
$$

Nas duas equaçóes apresentadas temos que $\mathrm{X}$ é a umidade em dado tempo; $t_{a d}=t \cdot N_{c} / X_{0}$, em que $t_{a d}$ é o tempo adimensional, $t$ é o tempo, $N_{c}$ é a taxa de secagem constante; TSN significa taxa de secagem normalizada $\left(\mathrm{N}_{\mathrm{T}} / \mathrm{N}_{\text {máx }}\right), \mathrm{N}_{\mathrm{T}}$ é a taxa instantânea de secagem e $\mathrm{N}_{\text {máx }}$ é a taxa máxima de secagem; $\mathrm{a}_{3}, \mathrm{a}_{4}, \mathrm{~b}_{3}$, $\mathrm{b}_{4}, \mathrm{e} \mathrm{c}_{4}$ são os parâmetros das equaçóes.

A cinética de secagem convectiva também

\begin{tabular}{|c|c|c|}
\hline MODELO & EQUAÇ̄̃o & REFERERNCIAS \\
\hline Exponencial Simples & $A D=\exp (-k t)$ & $\begin{array}{c}\text { Abe e Afzal } \\
\text { (1997) }\end{array}$ \\
\hline Page & $A D=\exp \left(-k t^{v}\right)$ & $\begin{array}{l}\text { Karathanos } \\
\text { e Belessiotis } \\
\text { (1999) }\end{array}$ \\
\hline Henderson e Pabis & $A D=A \exp (-k t)$ & $\begin{array}{l}\text { Henderson e } \\
\text { Pabis (1961) }\end{array}$ \\
\hline Logaritmo & $A D=B \exp (-k t)+c$ & $\begin{array}{c}\text { Yaldiz et al. } \\
(2001)\end{array}$ \\
\hline Exponencial dois termos & $\begin{array}{l}A D=C \exp \left(-k_{o} t\right) \\
+D \exp \left(-k_{1} t\right)\end{array}$ & $\begin{array}{c}\text { Ozdemir e } \\
\text { Devres (1999) }\end{array}$ \\
\hline Wang e Singh & $A D=1+E t+F t^{2}$ & $\begin{array}{c}\text { Wang e Singh } \\
\text { (1978) }\end{array}$ \\
\hline
\end{tabular}
pode ser caracterizada a partir dos dados do adimensional de umidade (AD) em função do tempo do processo. Os modelos descritos na Tabela 1 foram utilizados no ajuste aos dados experimentais.

Tabela 1 - Modelos matemáticos para a cinética de secagem

Em que: k, v, A, B, C, D, E, F, k e $_{1}$ são constantes dos modelos; t é o tempo de secagem (s).

Os parâmetros dos modelos cinéticos e de generalização foram obtidos por regressão não-li- 
near (Quasi-Newton), critério de convergência de 0,0001, com o auxílio do software Statistica $6.0^{\circ}$

O critério de escolha do melhor modelo para as generalizaçôes foi com base nos maiores valores do coeficiente de determinação $\left(\mathrm{R}^{2}\right)$ e do Teste $\mathrm{F}$, uma vez que estes parâmetros estatísticos já foram suficientes para se determinar o melhor modelo. Já para os modelos cinéticos, a escolha do melhor modelo foi baseada nos maiores valores do $\mathrm{R}^{2}$ e do Teste $\mathrm{F}$ e no menor valor da raiz do erro médio (RM). As Equaçóes 11 e 12 apresentam como foram calculados os valores do RM e do Teste F.

$$
\mathrm{RM}=\left[\frac{1}{\mathrm{~N}} \sum_{\mathrm{i}=1}^{\mathrm{N}}\left(\mathrm{X}_{\text {exp,i }}-\mathrm{X}_{\text {pred,i }}\right)^{2}\right]^{1 / 2}
$$

Teste $\mathrm{F}=\frac{\sum \overline{\mathrm{X}}_{\text {pred }}^{2}}{\sum \overline{\mathrm{X}}_{\text {resid }}^{2}}$

Em que: $\mathrm{X}_{\text {exp, } \mathrm{i}}$ é a umidade obtida experimentalmente, $X_{\text {pred,i }}$, a umidade predita pelo modelo, $X_{\text {resid, }}$ i, a umidade residual, definida como a diferença entre o $\mathrm{X}_{\text {exp,i }}$, e o $\mathrm{X}_{\text {pred,i }}$ e N, o número de experimentos.

\section{RESULTADOS E DISCUSSÃO}

A umidade inicial, em base seca, do bagaço do maracujá foi obtida em estufa a $105 \pm 3^{\circ} \mathrm{C}$ por $24 \mathrm{~h}$, sendo que o bagaço atingiu sua umidade de equilíbrio em aproximadamente $3 \mathrm{~h}$, mostrando, assim, uma secagem rápida característica de materiais que possuem bastante água livre. Nessa etapa concluiu-se que a água contida na matéria-prima representa $90,44 \%$ de sua massa total.

\subsection{CURVAS DE SECAGEM E DE TAXA DE SECAGEM}

Por meio das curvas de secagem e de taxa de secagem, foi possível analisar a influência da velocidade do fluxo de ar e da temperatura na secagem do bagaço do maracujá-amarelo. Na Figura 2 são mostradas as curvas de secagem obtidas para a velocidade do fluxo de ar de $1,3 \mathrm{~m} \cdot \mathrm{s}^{-1}$.
Figura 2 - Curvas de secagem para a velocidade do fluxo de ar de 1,3 m.s-1

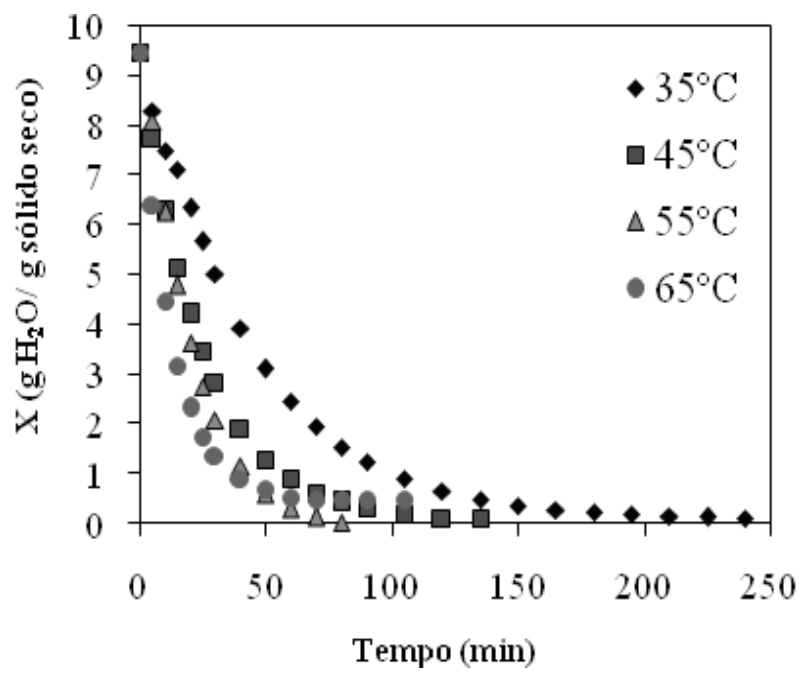

Pode-se observar pela Figura 2 que quanto maior a temperatura menor o tempo de secagem, uma vez que na velocidade do fluxo de ar de $1,3 \mathrm{~m} \cdot \mathrm{s}^{-1} \mathrm{e}$ temperatura de $35^{\circ} \mathrm{C}$ a amostra levou 225 min para atingir a umidade de equilíbrio, enquanto que na temperatura de $65^{\circ} \mathrm{C}$, o tempo de secagem foi de $120 \mathrm{~min}$.

A seguir estão apresentadas as curvas de taxa de secagem (R) para a avaliação da influência da temperatura no processo, obtidas a partir da derivação dos dados de umidade $(\mathrm{X})$ em relação ao tempo.

Figura 3 - Curvas de taxa de secagem para a velocidade do fluxo de ar de 1,3 m.s-1

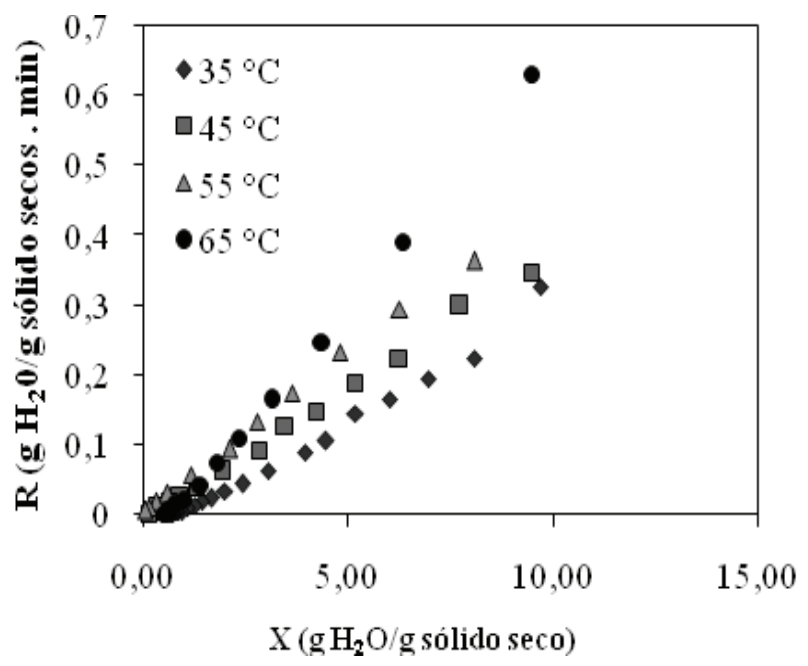

Observa-se a partir da Figura 3 que as curvas de taxa de secagem não apresentaram o período inicial de acondicionamento do material ao processo nem o período de taxa constante, apresentaram somente o período de taxa decrescente até se chegar a umidade de equilíbrio, comportamento este característico de cada material. Isto mostra que a difusão é o mecanismo físico dominante que rege o movimento da umidade nas amostras. 
Resultados semelhantes foram obtidos por Gogus e Maskan (1999) para a secagem do quiabo, Gupta et al. (2002) para a secagem do pimentão vermelho e Brizio et al. (2005) para a secagem da cebola que também apresentou elevada umidade inicial, cerca de $89,8 \%$ (b.u).

Verificou-se, também, que com o aumento da temperatura ocorre aumento da taxa de secagem, como pode ser verificado para as secagens nas temperaturas de 35 e $65^{\circ} \mathrm{C}$ e velocidade do fluxo de ar de $1,3 \mathrm{~m} \cdot \mathrm{s}^{-1}$, nas quais as taxas foram $0,33 \mathrm{~g} \mathrm{H}_{2} \mathrm{O}$. $\mathrm{min}^{-1}$ e $0,62 \mathrm{~g} \mathrm{H}_{2} \mathrm{O} \cdot \mathrm{min}^{-1}$, respectivamente.

$\mathrm{Na}$ Figura 4 é representado o mesmo tipo de gráfico, porém, para o ensaio a uma temperatura de $55^{\circ} \mathrm{C}$ e variando-se as velocidades do fluxo de ar.

Figura 4 - Taxa de secagem do bagaço do maracujá-amarelo em função da umidade na temperatura de $55^{\circ} \mathrm{C}$

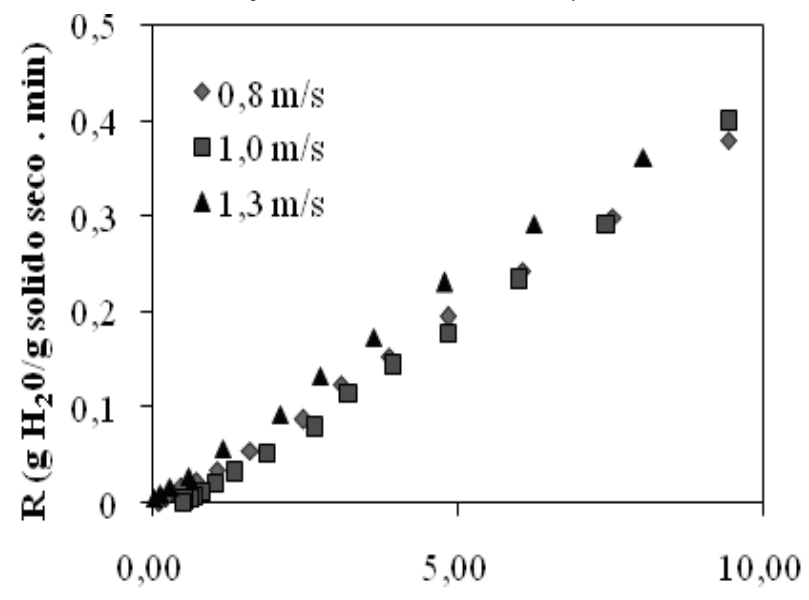

$\mathrm{X}\left(\mathrm{g} \mathrm{H}_{2} \mathrm{O} / \mathrm{g}\right.$ sólido seco)

Observando a Figura 4, nota-se que a velocidade do fluxo de ar não exerce influência significativa na secagem do bagaço do maracujá, diferentemente da temperatura, pois quanto maior a temperatura, maior o calor transferido ao material e consequentemente, a água presente no bagaço do maracujá migra mais facilmente para a superfície da mesma, facilitando a evaporação.

Para a determinaçáo da melhor condiçáo de trabalho, foi analisada cada condição de secagem. As temperaturas de $35^{\circ} \mathrm{C}$ e $45^{\circ} \mathrm{C}$ apresentaram maior umidade final e maior tempo de secagem em todas as velocidades do fluxo de ar, cerca de $4 \mathrm{~h}$ e $3 \mathrm{~h}$, respectivamente.

Já as temperaturas de $55^{\circ} \mathrm{C}$ e $65^{\circ} \mathrm{C}$ apresentaram menor umidade final e menor tempo de secagem, sendo que para ambas a umidade começa a ficar constante em torno de $80 \mathrm{~min}$ terminando o processo em 120 min para uma velocidade do fluxo de ar de $1,3 \mathrm{~m} \cdot \mathrm{s}^{-1}$, porém, à temperatura de $55^{\circ} \mathrm{C}$ gasta-se menos energia $(7700 \mathrm{~W})$ do que à temperatura $65^{\circ} \mathrm{C}(8500 \mathrm{~W})$. Por utilizar energia elétrica, este secador tem a vantagem de ser não poluente, apresentando uma elevada eficiência para este processo de secagem.

O mesmo comportamento foi verificado por Fiorentin (2009) para a secagem do bagaço de laranja em leito fixo, na qual a faixa de temperatura utilizada foi de 30 a $90^{\circ} \mathrm{C}$. A melhor condição de secagem obtida pelo autor foi para a temperatura de $45^{\circ} \mathrm{C}$ e velocidade do fluxo de ar de $1,3 \mathrm{~m} \cdot \mathrm{s}^{-1}$.

\subsection{COEFICIENTE DE DIFUSIVIDADE E DA ENERGIA DE ATIVAÇÃO}

Para a secagem do bagaço do maracujá-amarelo em camada delgada, o coeficiente de difusividade efetiva $\left(D_{\text {eff }}\right)$ foi calculado pela Equação 5, assumindo que a metade da espessura (L) das amostras foi de $0,005 \mathrm{~m}$. $A_{\text {eff }}$ foi determinada para cada condição de secagem estudada, conforme mostram as Figuras 5 a 7 .

Figura 5 - Efeito da temperatura na difusividade efetiva para velocidade do fluxo de ar de $0,8 \mathrm{~m} \cdot \mathrm{s}^{-1}$

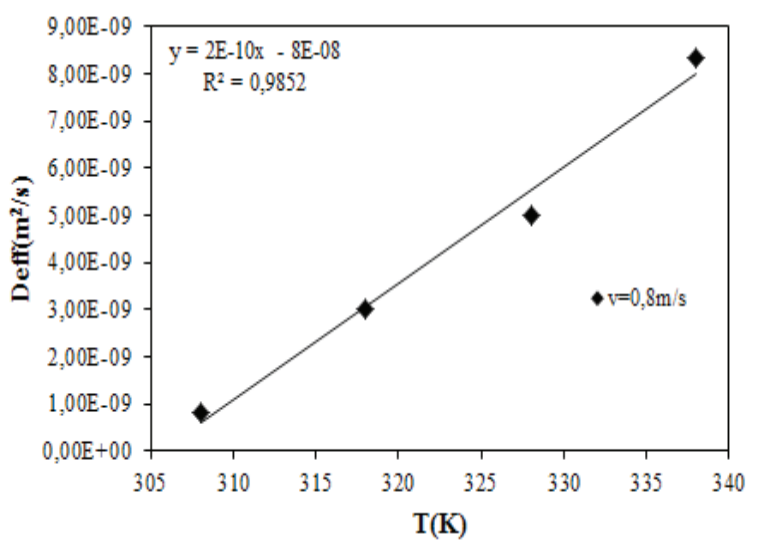

Figura 6 - Efeito da temperatura na difusividade efetiva para velocidade do fluxo de ar de 1,2 m.s.

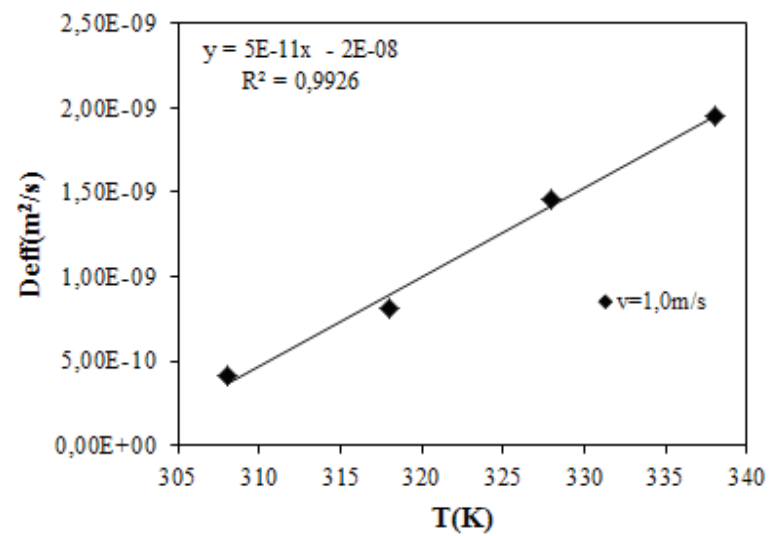


Figura 7 - Efeito da temperatura na difusividade efetiva para velocidade do fluxo de ar de 1,6 m.s-1

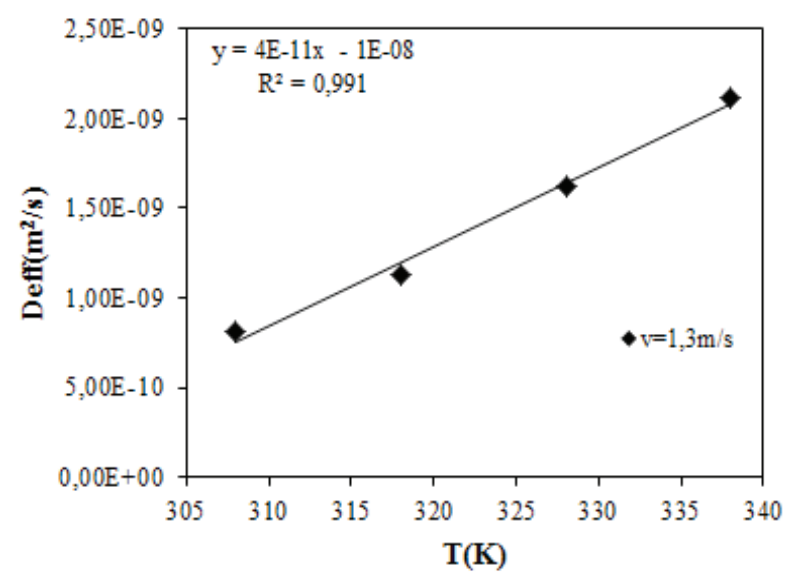

Pelas Figuras 5 a 7, pode-se observar um comportamento linear da difusividade em relação à temperatura para todas as velocidades do fluxo de ar testadas.

Os valores da difusividade para as temperaturas de $35,45,55$ e $65^{\circ} \mathrm{C}$ e velocidade do fluxo de ar de $1,3 \mathrm{~m} . \mathrm{s}^{-1}$ foram: $8,11.10^{-10}, 1,13.10^{-9}, 1,62.10^{-9}$ e $2,11.10^{-9} \mathrm{~m}^{2} / \mathrm{s}$, respectivamente, sendo que o mesmo comportamento foi obtido para os fluxos gasosos de 0,8 e 1,0 m.s. $\mathrm{s}^{-1}$. De acordo com os resultados, pode-se verificar que com o aumento da temperatura e velocidade do fluxo de ar ocorre também o aumento da difusividade efetiva, isto ocorre, pois a difusividade representa a velocidade com que a água migra do interior para a superfície do material, sendo então vaporizada. Segundo Madamba (2003) os valores do coeficiente de difusividade para materiais alimentícios encontram-se dentro de uma faixa de $10^{-11}$ a $10^{-9} \mathrm{~m}^{2} / \mathrm{s}$.

A energia de ativação foi determinada linearizando-se a Equação 6 e seus valores para as temperaturas de $35,45,55$ e $65^{\circ} \mathrm{C}$ e velocidade do fluxo de ar de 1,3 m.s.-1 foram: 7,706, 8,833, 10,093 e 11,143 $\mathrm{KJ} / \mathrm{mol}$, respectivamente. Fiorentin (2009) determinou a energia de ativaçáa para a secagem do bagaço de laranja, obtendo um valor de $10,669 \mathrm{KJ} / \mathrm{mol}$; Doymaz (2005) obteve uma energia de ativação de $28,36 \mathrm{KJ} / \mathrm{mol}$ para a secagem de cenoura e Park et al. (2002) obtiveram uma energia de ativaçáo de 82,93 $\mathrm{KJ} / \mathrm{mol}$ para a secagem de hortelá.

\subsection{CURVAS GENERALIZADAS DE SECAGEM E DE TAXA DE SECAGEM}

As generalizaçóes das curvas de secagem do bagaço do maracujá-amarelo para o intervalo de temperatura de 35 a $65^{\circ} \mathrm{C}$ e velocidades do fluxo de ar de $0,8,1,0$ e $1,3 \mathrm{~m} . \mathrm{s}^{-1}$ foram feitas por meio das Equaçóes 7, 8, 9 e 10 apresentadas anteriormente.
Assim, nas Figuras 8 e 10 são mostradas as curvas de secagem generalizadas conforme os modelos propostos por Page (1949) e Motta Lima et al. (2002). Já nas Figuras 9 e 11 são apresentados seus respectivos resíduos.

Figura 8 - Curva de secagem generalizada pelo modelo de Page (1949)

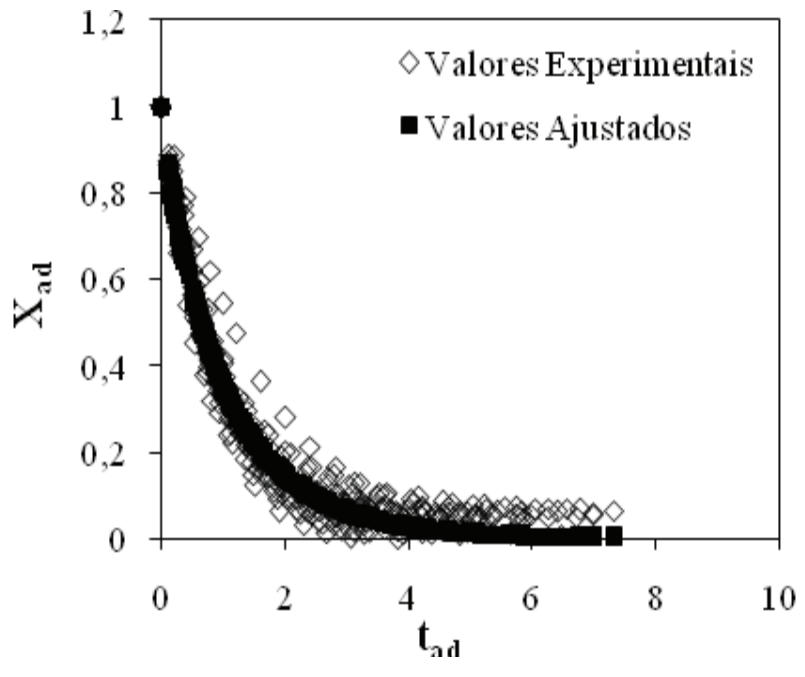

Figura 9 - Resíduo estimado para o modelo de Page (1949) na temperatura de $55^{\circ} \mathrm{C}$ e velocidade do ar de 1,3 m.s.

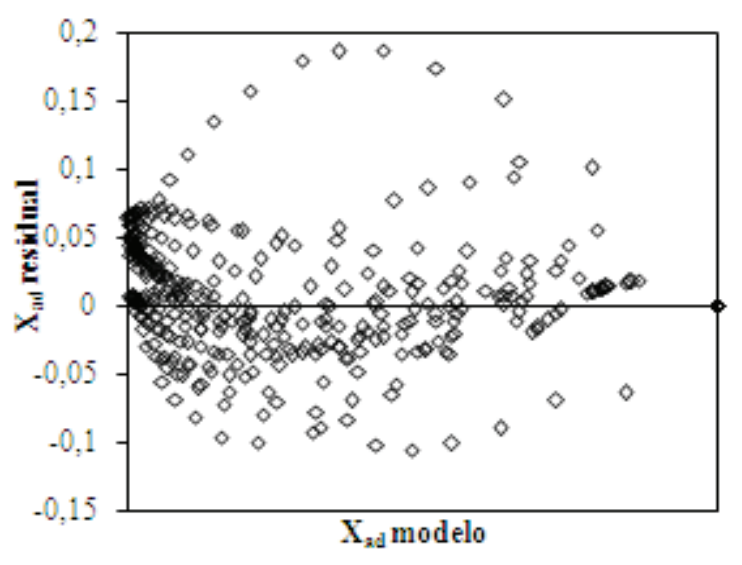

Figura 10 - Curva de secagem generalizada pelo modelo de Motta Lima et al. (2002)

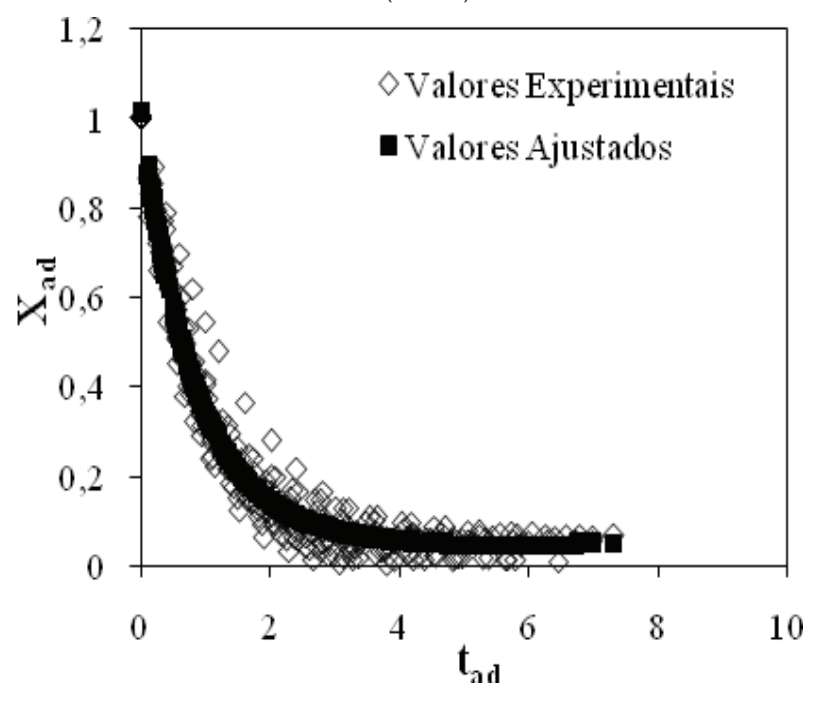


Figura 11 - Resíduo estimado para o modelo de Motta Lima et al. (2002) na temperatura de $55^{\circ} \mathrm{C}$ e velocidade do ar de 1,3 m.s-1

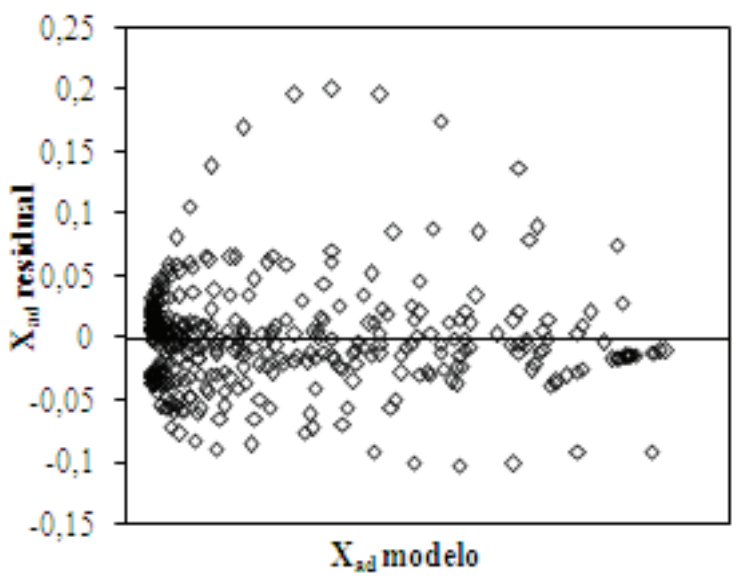

Pela análise das Figuras 8 e 10, pode-se observar que os modelos utilizados se ajustaram bem aos dados de secagem do bagaço do maracujá-amarelo.

Observa-se, nas Figuras 9 e 11, que os resíduos tiveram boa distribuiçáo aleatória em torno do zero, podendo ser verificada maior quantidade de pontos próximos à umidade de equilíbrio. Isto ocorre, pois a diferença entre a umidade do material e do ambiente, nesta regiáo, é muito pequena, o que faz com os resíduos sejam mais expressivos, não interferindo, desta maneira, na qualidade do ajuste, segundo Motta Lima (1999).

Os valores máximo e mínimo residuais foram de 0,1865 e -0,1049 para o modelo de Page (1949) e 0,2010 e -0,1029 para o modelo de Motta Lima et al. (2002).

As generalizações das curvas de taxa de secagem foram realizadas conforme os modelos propostos por Hogdes (1982) e Toffoli (2005) apresentados nas Figuras 12 e 14. Já nas Figuras 13 e 15 são apresentados seus respectivos resíduos.

Figura 12 - Curva de taxa de secagem generalizada pelo modelo de Hogdes (1982)

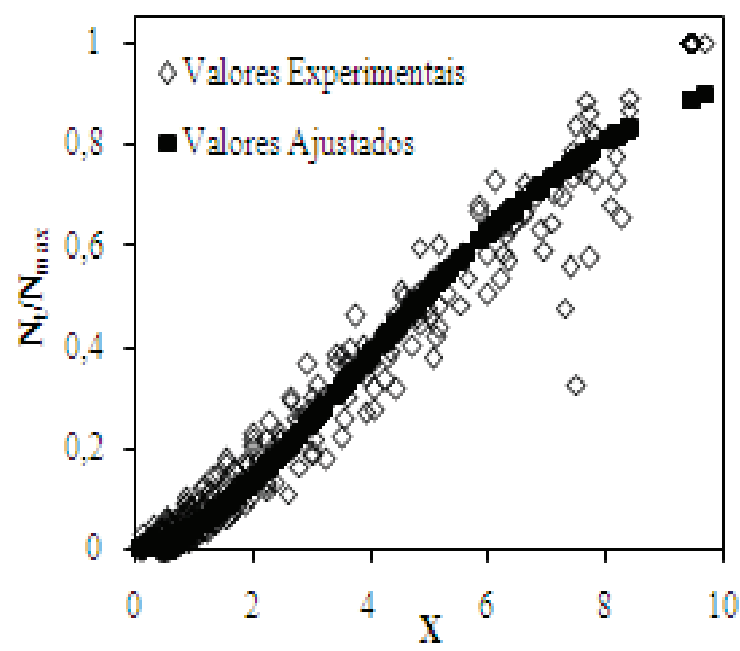

Figura 13 - Resíduo estimado para o modelo de Hogdes (1982) na temperatura de $55^{\circ} \mathrm{C}$ e velocidade do ar de $1,3 \mathrm{~m} \cdot \mathrm{s}^{-1}$

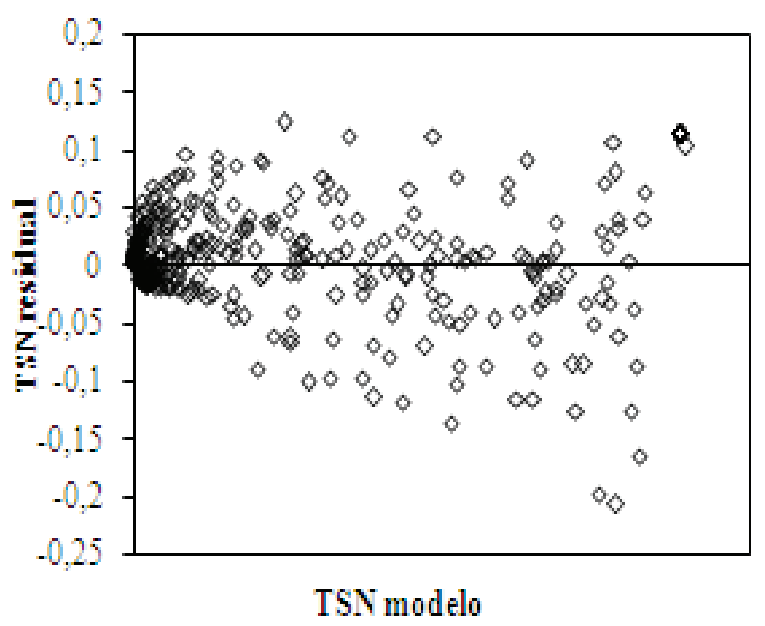

Figura 14 - Curva de taxa de secagem generalizada pelo modelo de Toffoli (2005)

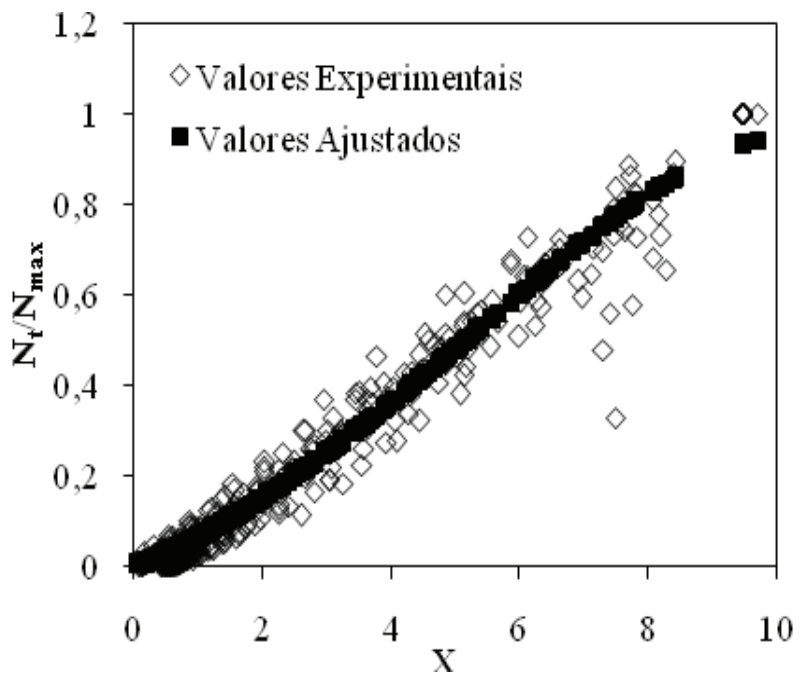

Figura 15 - Resíduo estimado para o modelo de Toffoli (2005) na temperatura de $55{ }^{\circ} \mathrm{C}$ e velocidade do ar de $1,3 \mathrm{~m} \cdot \mathrm{s}^{-1}$

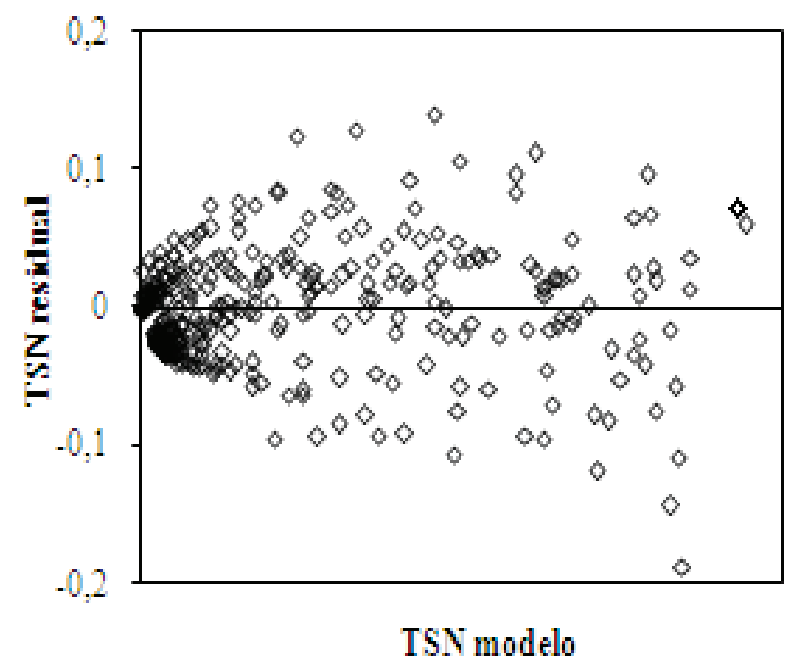


Por meio das Figuras 12 e 14 verificou-se que ambos os modelos utilizados na generalização das curvas de taxa de secagem se ajustaram bem aos dados experimentais.

Observa-se pelas Figuras 13 e 15 que os resíduos tiveram boa distribuiçáo aleatória em torno do zero, não apresentando nenhuma tendência específica, sendo que os valores máximo e mínimo residuais foram 0,1407 e - 0,1878 para o modelo de Toffoli (2005).

Os valores de $\mathrm{R}^{2}$, Teste $\mathrm{F}$ e dos parâmetros obtidos para os modelos em estudo encontram-se na Tabela 2.

Tabela 2 - Parâmetros obtidos na generalização das curvas de secagem e de taxa de secagem e valores do $R^{2}$ e Teste $F$

\begin{tabular}{l|c|c}
\hline \multicolumn{1}{c|}{ MODELO } & PARÂMETRO & VALOR \\
\hline \multirow{4}{*}{ Page } & $\mathrm{R}^{2}$ & 0,977 \\
\cline { 2 - 3 } & Teste $F$ & 90,362 \\
\cline { 2 - 3 } & $\mathrm{k} 1$ & 1,022 \\
\cline { 2 - 3 } & $\mathrm{a} 1$ & 0,910 \\
\hline \multirow{4}{*}{ Motta Lima et al. (2002) } & $\mathrm{R}^{2}$ & 0,981 \\
\cline { 2 - 3 } & Teste F & 110,192 \\
\cline { 2 - 3 } & $\mathrm{k} 2$ & 1,094 \\
\cline { 2 - 3 } & $\mathrm{a} 2$ & $-0,065$ \\
\hline \multirow{4}{*}{ Hogdes } & $\mathrm{b} 2$ & 1,021 \\
\cline { 2 - 3 } & $\mathrm{R}^{2}$ & 0,963 \\
\cline { 2 - 3 } & Teste F & 46,499 \\
\cline { 2 - 3 } & $\mathrm{a} 3$ & 6,080 \\
\cline { 2 - 3 } & $\mathrm{b} 3$ & 1,760 \\
\hline \multirow{4}{*}{ Toffoli } & $\mathrm{R}^{2}$ & 0,969 \\
\cline { 2 - 3 } & Teste F & 55,015 \\
\cline { 2 - 3 } & $\mathrm{a} 4$ & 6,270 \\
\cline { 2 - 3 } & $\mathrm{b} 4$ & 0,101 \\
\cline { 2 - 3 } & $\mathrm{c} 4$ & 0,104 \\
\hline
\end{tabular}

Observa-se por meio da Tabela 2 que o modelo de Motta Lima et al. (2002) conseguiu melhor ajuste aos dados experimentais, apresentando valores de $\mathrm{R}^{2}$ e do Teste $\mathrm{F}$ superiores aos do modelo de Page (1949). O mesmo comportamento foi observado para o modelo de Toffoli (2005) em relação ao de Hogdes (1982).
Os resultados obtidos estão de acordo com Fiorentin (2009), que também aplicou estes modelos de curvas generalizadas de secagem e de taxa de secagem na análise da cinética de secagem do bagaço da laranja.

A aplicação dos modelos matemáticos para a cinética de secagem apresentados na Tabela 1 foi realizada para cada condição de secagem. A Figura 16 apresenta o comportamento dos modelos matemáticos com base nos dados experimentais para a secagem a $55^{\circ} \mathrm{C}$ e velocidade do fluxo de ar de $1,3 \mathrm{~m} \cdot \mathrm{s}^{-1}$.

Figura 16 - Ajustes de modelos matemáticos aos dados de secagem na temperatura de $55^{\circ} \mathrm{C}$ e velocidade do ar de $1,3 \mathrm{~m} \cdot \mathrm{s}^{-1}$

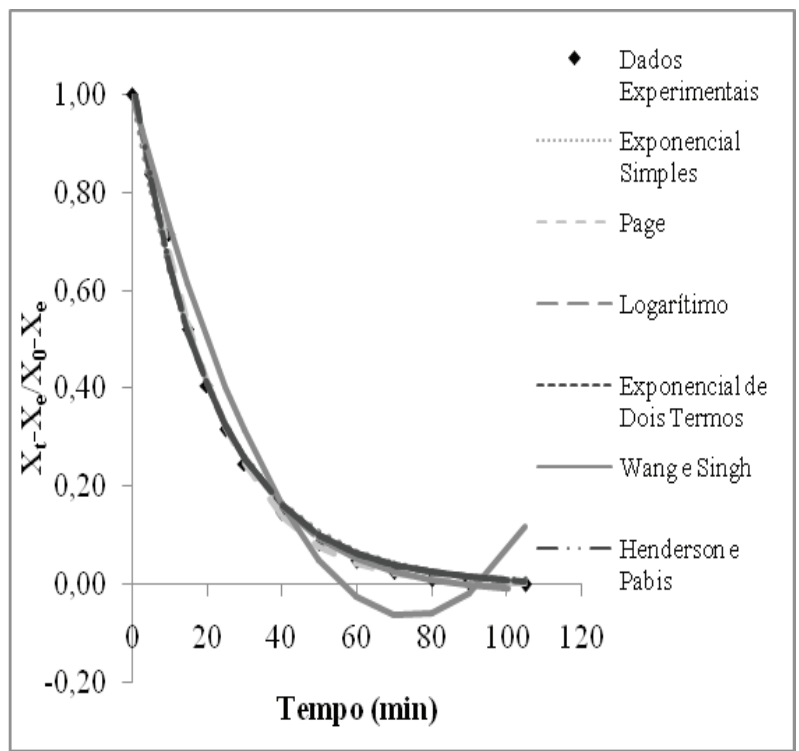

Por meio da Figura 16, observa-se que, exceto o modelo de Wang e Singh (1978), os modelos utilizados apresentam boa relaçáo com os dados experimentais, não sendo possível ser determinado qual o melhor modelo, apenas observando-se o gráfico. Esse resultado foi obtido também nos outros ensaios. Sendo assim, o melhor modelo foi escolhido com base nos maiores valores do coeficiente de determinação $\left(\mathrm{R}^{2}\right)$ e do Teste $\mathrm{F}$, e no menor valor da raiz do erro médio (RM). 
Tabela 3 - Comparação do comportamento dos modelos e parâmetros obtidos nos ajustes dos modelos matemáticos para a cinética de secagem do bagaço do maracujá-amarelo

\begin{tabular}{|c|c|c|}
\hline MODELO & PARÂMETRO & VALOR \\
\hline \multirow{4}{*}{ Exponencial Simples } & $R^{2}$ & 0,9936 \\
\hline & Teste F & 287,620 \\
\hline & RM & 0,0258 \\
\hline & k & 0,0447 \\
\hline \multirow{5}{*}{ Page } & $R^{2}$ & 0,9986 \\
\hline & Teste F & 1396,841 \\
\hline & RM & 0,0120 \\
\hline & k & 0,0260 \\
\hline & $\nu$ & 1,1723 \\
\hline \multirow{5}{*}{ Henderson e Pabis } & $R^{2}$ & 0,9952 \\
\hline & Teste F & 405,545 \\
\hline & RM & 0,0223 \\
\hline & $k$ & 0,0466 \\
\hline & $\mathrm{a}$ & 1,0385 \\
\hline \multirow{6}{*}{ Logarítmo } & $R^{2}$ & 0,9961 \\
\hline & Teste F & 493,612 \\
\hline & RM & 0,0202 \\
\hline & $\mathrm{a}$ & 1,0519 \\
\hline & k & 0,0442 \\
\hline & $c$ & $-0,0200$ \\
\hline \multirow{7}{*}{$\begin{array}{l}\text { Exponencial de dois } \\
\text { Termos }\end{array}$} & $R^{2}$ & 0,9952 \\
\hline & Teste F & 405,545 \\
\hline & RM & 0,0223 \\
\hline & $a$ & 0,5192 \\
\hline & $\mathrm{k} 0$ & 0,0466 \\
\hline & $b$ & 0,5193 \\
\hline & k1 & 0,0466 \\
\hline \multirow{5}{*}{ Wang e Singh } & $R^{2}$ & 0,9546 \\
\hline & Teste F & 48,336 \\
\hline & RM & 0,0689 \\
\hline & $\mathrm{a}$ & $-0,028661$ \\
\hline & $b$ & 0,000193 \\
\hline
\end{tabular}

Por meio da Tabela 3, verifica-se que o modelo de Page apresentou os maiores valores de $\mathrm{R}^{2}$ e do Teste F e o menor valor do RM, mostrando, assim, o seu elevado potencial de representaçáo dos dados experimentais em todas as condiçóes de secagem estudadas. O modelo Exponencial de Dois Termos e de Henderson e Pabis apresentaram a mesma tendência em relação aos valores do Teste F, RM e R².

Os resultados obtidos estáo de acordo com Orikasa et al. (2008); Kingsly e Singh (2007); Martinazzo et al. (2007); Mwithiga e Olwal (2005); Doymaz (2005), que avaliaram o comportamento da secagem de alimentos em função do tempo e obtiveram resultados semelhantes.

\section{CONCLUSÃO}

Para o processo de secagem do bagaço, verificou-se que na temperatura de $55^{\circ} \mathrm{C}$ e velocidade do ar de $1,3 \mathrm{~m} \cdot \mathrm{s}^{-1}$ a umidade de equilíbrio foi atingida mais rapidamente com economia de tempo e energia, quando comparada com as demais condiçóes estudadas, sendo esta então, a melhor condição de secagem.

As curvas generalizadas de secagem obtidas na faixa de temperatura de 35 a $65^{\circ} \mathrm{C}$ mostraram que o modelo de Motta Lima et al. (2002) apresentou melhor ajuste aos dados experimentais com base nos valores de $\mathrm{R}^{2}$ e do Teste F. Para as curvas generalizadas de taxa de secagem, o modelo de Toffoli (2005) foi o que melhor descreveu os dados experimentais.

A aplicação dos modelos matemáticos aos dados experimentais de secagem mostrou que o modelo de Page (1949) foi o que melhor representou os dados experimentais em todas as condiçóes estudadas.

$\mathrm{O}$ número de parâmetros envolvidos no projeto de secadores exige uma modelagem mesmo que empírica para se reduzir o trabalho de engenharia envolvido no dimensionamento das unidades de processamento.

\section{REFERÊNCIAS}

Abe, T.; Afzal, T. M. Thin-layer in infrared radiation drying of rough rice. Journal of Agricultural Engineering Research, v. 67, p. 289-297, 1997.

Bernacci, L. C.; Meletti, L. M. M.; Soares-Scott M. D.; Passos I. R. S.; Junqueira N. T. V. Espécies de maracujá: caracterização e conservação da biodiversidade. In: Faleiro FG; Junqueira NTV; Braga MF (Eds). Maracujá: germoplasma e melhoramento genético. Planaltina-DF: Embrapa Cerrados. 2005. p.559-586.

Brizio, A. P. R.; Weska, R. F.; Rizzi, J.; PINTO, L. A. A. Secagem de cebola (Allium cepa L.) em leito fixo utilizando escoamento de ar reverso. In: Anais do VI COBEQ IC, Universidade Estadual de Campinas, 2005.

Corrêa, P. C.; Ribeiro, D. M.; Resende, O.; Botelho, F. M. Determinação e modelagem das propriedades fisicas e da contração volumétrica do trigo, durante a secagem. Revista Brasileira de Engenharia Agrícola e Ambiental, v.10, n.3, p.665-670, 2006.

CRONQUIST, A. An integrated system of classification of flowering plants. New York: Columbia University, p.1262, 1981. 
Doymaz, I. Air-drying characteristics of tomatoes. Journal of Food Engineering, v.78, p.1291-1297, 2005.

Ferrari, R. A.; Colussi, F.; Ayub, R. A. Caracterização de subprodutos da industrialização do maracujá: aproveitamento das sementes. Revista Brasileira de Fruticultura, 26(1), p. 101-102, 2004.

Fiorentin, L. D. Remoção de Corante de Efluentes da Indústria Têxtil Utilizando Processos com Membranas e Adsorção em Bagaço de Laranja. Tese (Doutorado), Universidade Estadual de Maringá, Maringá-PR, 2009.

Foust, A. S., Wenzel, L. A.; Clump, C. W. Principles of Unit Operations, 2nd Ed., John Wiley \& Sons Inc., 1980.

Gogus, F.; Maskan, M. Water adsorption and drying characteristics of okra (Hibiscus Esculentus L.). Drying Technology, v.17, p.883-894,1999.

Gupta, P.; Ahmed, J.; Shivhare, U. S.; Raghavan, G. S. V. Drying characteristics of red chilli. Drying Technology, v.20, p. 1975-1987, 2002.

Henderson, S. M.; Pabis, S. Grain drying theory I: temperature effect on drying coefficient. Journal of Agricultural Engineering Research, v. 6, p.169-174, 1961.

Hogdes, C. R. Laboratory drying study and applications for paper machine drying. Hemisphere, p. 99-105, 1982.

Karathanos, V.T.; Belessiotis, V.G. Application of a thin-layer equation to drying data of fresh and semi-dried fruits. Journal of Agricultural Engineering Research, v. 74, p. 355-36, 1999.

Kingsly, A. R. P.; Singh, D. B. Drying kinetics of pomegranate arils. Journal of Food Engineering, v. 79, p. 741-744, 2007.

Luz, G. R. Modelagem matemática e análise do secador rotativo de farelo de soja. Dissertação (Mestrado), Universidade Estadual de Maringá, Maringá-PR, 2006.

Madamba, P. S. Thin layer drying models for osmotically pré-dried young coconut. Drying technology, v.21, n.9, p.1759-1780, 2003.
Martinazzo, A. P.; Corrêa, P. C.; Resende, O.; Melo, E. C. Análise e descrição matemática da cinética de secagem de folhas de capim-limão. Revista Brasileira de Engenharia Agrícola e Ambiental, v.11, n.3, p. 301-306, 2007.

Motta Lima, O. C.; Pereira, N. C.; Mendes, E. S. Curvas generalizadas na análise da cinética de secagem de papel artesanal com ar ambiente em conveç̧âo forçada. In: Anais em CD-Rom do XXX Congresso Brasileiro de Sistemas Particulados, Universidade Federal de Sáo Carlos, 2002.

Motta Lima, O. C. Estudo sobre a secagem de folhas de celulose. Tese (Doutorado), Universidade Federal do Rio de Janeiro, Rio de Janeiro-RJ, 1999.

Mwithiga, G.; Olwal, J. O. The drying kinetics of kale (brassica oleracea) in a convective hot air dryer. Journal of Food Engineering, v.71, p.373-378, 2005.

Orikasa, T.; Wub, L.; Shiina, T.; Tagawa, A. Drying characteristics of kiwifruit during hot air drying. Journal of Food Engineering, v. 85, p. 303-308, 2008.

Ozdemir, M.; Devres, Y. O. The thin-layer drying characteristics of hazelnuts during roasting. Journal of Food Engineering, v. 42, p. 225-233, 1999.

Page, G. E. Factors influencing the maximum rates of air drying shelled corn in thin layer. Thesis (Master of Science) - Purdue University, 1949.

Toffoli, C.M. Análise da influência das varáveis do processo de secagem no coeficiente de contato térmico entre o papel e cilindro. Dissertaçáo (Mestrado), Universidade Estadual de Maringá, Maringá-PR, 2005.

Zanoelo, E. F.; Celso, G. M. D.; Kaskantzis. Drying kinetics of mate leaves in a packed bed dryier. Byosystems Engineering, v.96, n.4, p.487-494, 2007.

Yaldiz, O.; Ertekin, C.; Uzun, H.I. Mathematical modeling of thin-layer solar drying of sultana grapes. Energy, v. 26, p. 457-465, 2001.

Wang, C.Y.; Singh, R.P. Use of variable equilibrium moisture content in modeling rice drying. ASAE Paper, $\mathrm{p}$. 78-6505, ASAE, St. Joseph-MI, 1978. 